\title{
The effect of Hall current on an unsteady MHD free convective viscous dissipative chemically reacting fluid flow along a vertical porous plate with thermal radiation
}

\author{
Mamta Thakur \& M. Venkata Krishna \\ Department of Mathematics, University College of Science, Osmania University, Hyderabad, 500007, Andhra \\ Pradesh, India.
}

\begin{abstract}
The effect of hall current onan unsteady magnetohydrodynamic flow of heat and mass transfer of an electrically conducting incompressible fluid along an infinite vertical porous plate in presence ofthermal radiation, viscous dissipationand chemical reaction has been studied.The fluid is considered to be a gray, absorbing emitting but non - scattering medium and the Rosseland approximation is used to describe the radiative heat flux in the energy equation. The dimensionless governing coupled, non - linear boundary layer partial differential equations are solved by an efficient finite element method forvelocity, temperature, concentration field and also the expression for shearing stress, Rate of heat and mass transferare obtained. The effect of thermal Grashof Number, solutal Grashof Number, Transpiration cooling parameter, Prandtl Number, Schmidt Number,Hartmann number, Thermal radiation parameter, Eckert number, Hall parameterand Chemical reaction parameter on the flow field are shown through graphs and tables.The numerical results for some special cases were compared with Sriramuluet al. [19] and were found to be in good agreement. This present problem has wide applications in several areas of science and technology viz. chemical catalytic reactors, thermal insulators, heat exchanger devices, nuclear waste repositories, drying of porous solids, enhanced oil and gas recovery, underground energy transport etc.
\end{abstract}

Key words: Hall current,MHD flow, Free convection, Thermal radiation, Viscous dissipation, Chemical reaction, Galerkin finiteelement method.

$C^{\prime} \quad$ Dimensionless concentration

$\varepsilon \quad$ Porosity of the porous medium

$C_{w}^{\prime} \quad$ Concentration near the plate

$C_{\infty}^{\prime} \quad$ Concentration in the fluid far

$\theta$ Dimensionless Temperatureaway from the plate

$T^{\prime} \quad$ Temperature of the fluid

$T_{w}^{\prime} \quad$ Temperature of the plate

$T_{\infty}^{\prime} \quad$ Temperature of the fluid far away from the plate

$u^{\prime} \quad$ Velocity component in $x^{\prime}$ direction

$w^{\prime} \quad$ Velocity component in $z^{\prime}-$ direction

$x^{\prime} \quad$ Spatial co - ordinate along the plate

$v \quad$ Kinematics viscosity, $\mathrm{m}^{2} /$ splate

$y^{\prime}$ Spatial co - ordinate normal to the plate

$\alpha \quad$ Thermal Diffusivity

$k_{e} \quad$ Mean absorption coefficient

$\bar{V} \quad$ Velocity vector, $\mathrm{m} / \mathrm{s}$

$\kappa \quad$ Thermal conductivity, W/mK

$m \quad$ Hall parameter

\section{Nomenclature:}

$\sigma \quad$ Electrical conductivity, mho/m

$\mu \quad$ Viscosity, $\mathrm{Ns} / \mathrm{m}^{2}$

$\mu_{e} \quad$ Magnetic permeability,

Henry/meter

$C_{p} \quad$ Specific heat at constant Pressure,

$\mathrm{J} / \mathrm{kg}-\mathrm{K}$

$\rho \quad$ Density, $\mathrm{kg} / \mathrm{m}^{3}$

$\omega_{e}$ Electron frequency, radian/sec

$D$ Chemical molecular diffusivity

$\tau_{e} \quad$ Electron collision time in Sec

$U_{o} \quad$ Reference velocity

$e \quad$ Electron charge, coulombs

$M \quad$ Hartmann number

$n_{e} \quad$ Number density of the electron

Pr Prandtl number

$P_{e} \quad$ Electron Pressure, $\mathrm{N} / \mathrm{m}^{2}$

Sc Schmidt Number

$g$ Acceleration due to Gravity, 9.81 $\mathrm{m} / \mathrm{s}^{2}$

$G r$ thermal Grashof Number $\quad \beta$

Volumetric co - efficient of thermal Expansion, $\mathrm{K}^{-1}$

Gc solutal Grashof Number 


$\begin{array}{ll}R & \text { Thermal radiation parameter } \\ E c & \text { Eckert number } \\ k_{r} & \text { Chemical reaction parameter }\end{array}$

\author{
$\beta^{*} \quad$ Co - efficient of volume expansion with \\ Species concentration \\ $\lambda$ Non-dimensional transpiration parameter
}

\section{Introduction:}

Considerable attention has been given to the unsteady free convection flow of viscous incompressible, electrically conducting fluid in presence of applied magnetic field in connection with the theories of fluid motion in the liquid core of the Earth and also meteorological and oceanographic applications. In recent years, the analysis of hydromagnetic flow involving heat and mass transfer in porous medium has attracted the attention of many scholars because of its possible applications in diverse fields of science and technology such as - soil sciences, astrophysics, geophysics, nuclear power reactors etc. In geophysics, it finds its applications in the design of MHD generators and accelerators, underground water energy storage system etc. It is worth mentioning that MHD is now undergoing a stage of great enlargement and differentiation of subject matter. These new problems draw the attention of the researchers due to their varied significance, in liquid metals, electrolytes and ionized gases etc. In addition, the applications of the effect of Hall current on the fluid flow with variable concentration have been seen in MHD power generators, astrophysical and meteorological studies as well as in plasma physics. The Hall Effect is due merely to the sideways magnetic force on the drifting free charges. The electric field has to have a component transverse to the direction of the current density to balance this force. In many works on plasma physics, the Hall Effect is disregarded. But if the strength of magnetic field is high and the number density of electrons is small, the Hall Effect cannot be ignored as it has a significant effect on the flow pattern of an ionized gas. Hall Effect results in a development of an additional potential difference between opposite surfaces of a conductor for which a current is induced perpendicular to both the electric and magnetic field. This current is termed as Hall current. Model studies on the effect of Hall current on MHD convection flows have been carried out by many authors due to application of such studies in the problems of MHD generators and Hall accelerators.

From the point of view of applications, this effect can be taken into account within the range of magnetohydrodynamical approximation. Abdul Maleque et al. [1] studied the effects of variable properties and Hall current on steady MHD laminar convective fluid flow due to a porous rotating disk. Ajay Kumar Singh [2] made an attempt to study the steady MHD free convection and mass transfer flow with hall current, viscous dissipation and joule heating, taking in to account the thermal diffusion effect. Anand Rao et al. [3] investigated applied magnetic field on transient free convective flow of an incompressible viscous dissipative fluid in a vertical channel. Anjali Devi et al. [4] discussed pulsated convective MHD flow with Hall current, heat source and viscous dissipation along a vertical porous plate. Atul Kumar Singhet al. [5] studied hydromagnetic free convection and mass transfer flow with Joule heating, thermal diffusion, Heat source and Hall current. Chowdhary et al. [6] studied heat and mass transfer in elasticoviscous fluid past an impulsively started infinite vertical plate with Hall Effect. Chaudhary et al. [7] analyzed Hall Effect on MHD mixed convection flow of a viscoelastic fluid past an infinite vertical porous plate with mass transfer and radiation. On the effectiveness of viscous dissipation and Joule heating on steady MHD and slip flow of a Bingham fluid over a porous rotating disk in the presence of Hall and ion - slip currents was studied by Emmanuel Osalusi et al. [8]. Hossainet al.[9]investigated the effect of hall current on the unsteady freeconvection flow of a viscous incompressible fluid with heat and mass transfer along a vertical porous plate subjected to a time dependent transpiration velocity when the constant magnetic field is applied normal to the flow. Lai [10] studied the coupled heat and mass transfer by mixed convection from vertical plate in a saturated porous medium. Maleque et al. [11] investigated the effects of variable properties and hall current on steady MHD laminar convective fluid flow due to a porous rotating disk. MHD stationary symmetric flows withHall effect was analyzed by Palumbo et al. [12]. Raja Shekhar et al. [13] studied the effect of hall current on free convection and mass transfer flow through a porous medium bounded by an infinite vertical porous plate, when the plate was subjected to a constant suction velocity and heat flux. Singh et al. [14] studied the effects of mass transfer on the flow past a vertical porous plate. Singh et al. [15] studied an integral treatment for combined heat and mass transfer by natural convention in a porous medium. Singhet al. [16] analyzed the free convection heat and mass transfer along a vertical surface in a porous medium. Later, Soundalgekar et al. [17] studied the coupled heat and mass transfer by natural convection from vertical surfaces in a porous medium.Hall Effect on MHD Flow and Heat Transfer along a Porous Flat Plate with Mass Transfer and Source/Sink was analyzed by Srihari et al. [18]. Sriramulu et al. [19] discussed the effect of Hall Current on MHD flow and heat transfer along a porous flat plate with mass transfer.

In this paper we studied theHall Effect on MHD flow and mass transfer of an electrically conducting incompressible fluid along an infinite vertical porous plate with thermal radiation, viscous dissipation and chemical reaction.Also, the effects of different flow parameters encountered in the equations are studied. The 
results obtained are good agreement with the results of Sriramuluet al. [19]. Theproblem is governed by system of Coupled Non - linear partial differential equations whose exact solution is difficult to obtain. Hence, the problem is solved by using Galerkin finite element method, which is more economical from computational view point.

\section{Mathematical formulation:}

An unsteady free convection flow of an electrically conducting viscous incompressible fluid with mass transfer along a porous flat plate has been considered with viscous dissipation. The flow is assumed to be in $x^{\prime}$ - direction, which is taken along the plate in upward direction. The $y^{\prime}$ - direction which is taken along the normal to the direction of the plate. Initially,for time $t^{\prime} \leq 0$, the plate and the fluid are maintained at the same constant temperature $T_{\infty}^{\prime}$ in a stationary condition with the same species concentration $C_{\infty}^{\prime}$ at all points. When $t^{\prime}>0$, the temperature of the plate is instantaneously raised (or lowered) to $T_{w}^{\prime}$ and the concentration of the species is raised (or lowered) to $C_{w}^{\prime}$, which are hereafter regarded as constant.A magnetic field of uniform strength is assumed to be applied transversely to the porous plate. The magnetic Reynolds number of the flow is taken to be small enough so that the induced magnetic field can be neglected.

Using the relation $\nabla \cdot \bar{H}=0$ for the magnetic field $\bar{H}=\left(H_{x}, H_{y}, H_{z}\right)$, we obtain $H_{y}=$ constant $=$ $H_{o}$ (say)where $H_{o}$ is the externally applied transverse magnetic field so that $\bar{H}=\left(0, H_{o}, 0\right)$. The equation of conservation of electric charge $\nabla . \bar{J}=0$ gives $j_{y}=$ constant, where $\bar{J}=\left(j_{x}, j_{y}, j_{z}\right)$. We further assume that the plate is non - conducting. This implies $j_{y}=0$ at the plate and hence zero everywhere. When the strength of magnetic field is very large,the generalized Ohm's law in the absence of electric field takes the following form:

$$
\bar{J}+\frac{\omega_{e} \tau_{e}}{B_{o}} \bar{J} \times \bar{H}=\sigma\left(\mu_{e} \bar{V} \times \bar{H}+\frac{1}{e n_{e}} \nabla P_{e}\right)
$$

Under the assumption that the electron pressure (for weakly ionized gas), the thermo - electric pressure and ion - slip conditions are negligible, equation (1) becomes:

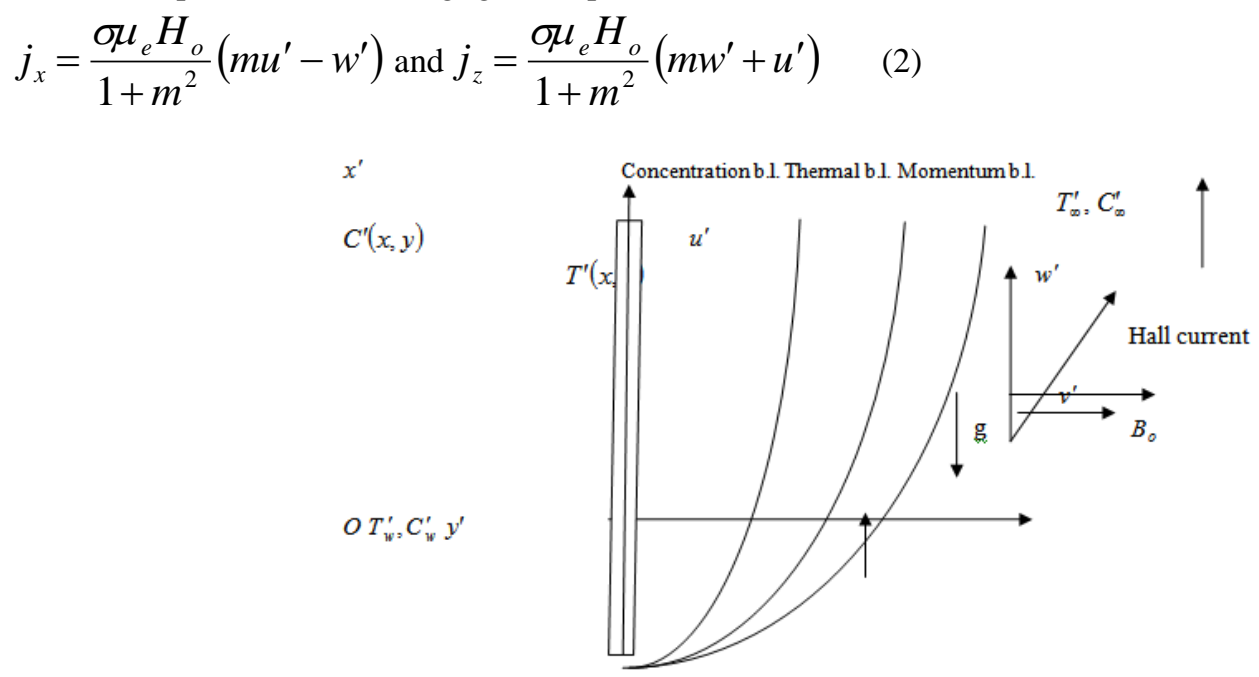

Figure 1: The coordinate system for the physical model of the problem.

Where $u^{\prime}$ is the $x^{\prime}$ - component of $\bar{V}, w^{\prime}$ is the $z^{\prime}-$ component of $\bar{V}$ and $m\left(=\omega_{e} \tau_{e}\right)$ is the hall parameter. Within the above framework, the equations which govern the flow under the usual Boussinesq's approximation are as follows:

$$
\begin{aligned}
& \frac{\partial v^{\prime}}{\partial y^{\prime}}=0(3) \frac{\partial u^{\prime}}{\partial t^{\prime}}+v^{\prime} \frac{\partial u^{\prime}}{\partial y^{\prime}}=v \frac{\partial^{2} u^{\prime}}{\partial y^{\prime 2}}-\frac{\sigma \mu_{e}^{2} H_{o}^{2}}{\rho\left(1+m^{2}\right)}\left(u^{\prime}+m w^{\prime}\right)+g \beta\left(T^{\prime}-T_{\infty}^{\prime}\right)+g \beta^{*}\left(C^{\prime}-C_{\infty}^{\prime}\right)(4) \\
& \frac{\partial w^{\prime}}{\partial t^{\prime}}+v^{\prime} \frac{\partial w^{\prime}}{\partial y^{\prime}}=v \frac{\partial^{2} w^{\prime}}{\partial y^{\prime 2}}-\frac{\sigma \mu_{e}^{2} H_{o}^{2}}{\rho\left(1+m^{2}\right)}\left(w^{\prime}-m u^{\prime}\right)_{(5)}
\end{aligned}
$$




$$
\begin{aligned}
& \frac{\partial T^{\prime}}{\partial t^{\prime}}+v^{\prime} \frac{\partial T^{\prime}}{\partial y^{\prime}}=\frac{\kappa}{\rho c_{p}} \frac{\partial^{2} T^{\prime}}{\partial y^{\prime 2}}+\frac{v}{c_{p}}\left(\frac{\partial u^{\prime}}{\partial y^{\prime}}\right)^{2}-\frac{\partial q_{r}}{\partial y^{\prime}}(6) \\
& \frac{\partial C^{\prime}}{\partial t^{\prime}}+v^{\prime} \frac{\partial C^{\prime}}{\partial y^{\prime}}=D \frac{\partial^{2} C^{\prime}}{\partial y^{\prime 2}}-K_{r}^{\prime} C^{\prime}
\end{aligned}
$$

The initial and boundary conditions of the problem are:

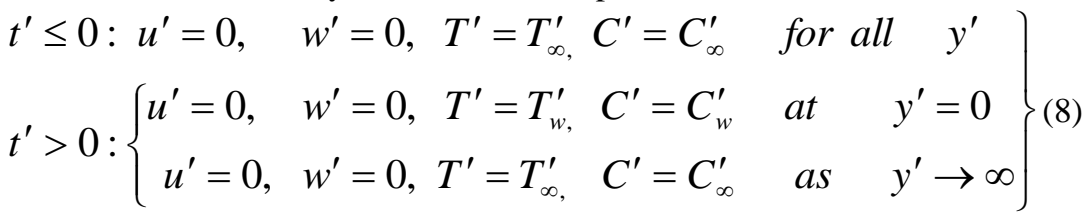

The radiative heat flux term is simplified by making use of the Rosseland approximation [20] as $q_{r}=-\frac{4 \bar{\sigma}}{3 k^{*}} \frac{\partial T^{4}}{\partial y^{\prime}}$

Here $\bar{\sigma}$ is Stefan - Boltzmann constant and $k^{*}$ is the mean absorption coefficient. It is assumed that the temperature differences within the flow are sufficiently small so that $T^{\prime 4}$ can be expressed as a linear function of $T^{\prime}$ after using Taylor's series to expand $T^{\prime 4}$ about the free stream temperature $T_{h}^{\prime}$ and neglecting higher - order terms. This results in the following approximation:

$T^{\prime 4} \cong 4 T_{h}^{\prime 3} T^{\prime}-3 T_{h}^{\prime 4}$

Using equations (9) and (10) in the last term of equation (6), we obtain:

$\frac{\partial q_{r}}{\partial y}=-\frac{16 \bar{\sigma} T_{h}^{\prime 3}}{3 k^{*}} \frac{\partial^{2} T^{\prime}}{\partial y^{\prime 2}}$

Introducing (8) in the equation (3), the energy equation becomes:

$\frac{\partial T^{\prime}}{\partial t^{\prime}}+v^{\prime} \frac{\partial T^{\prime}}{\partial y^{\prime}}=\frac{\kappa}{\rho c_{p}} \frac{\partial^{2} T^{\prime}}{\partial y^{\prime 2}}+\frac{v}{c_{p}}\left(\frac{\partial u^{\prime}}{\partial y^{\prime}}\right)^{2}+\frac{16 \bar{\sigma} T_{h}^{\prime 3}}{3 k^{*}} \frac{\partial^{2} T^{\prime}}{\partial y^{\prime 2}}(12)$

The non - dimensional quantities introduced in the equations (4), (5) and (12) are:

$$
\begin{aligned}
& t=\frac{t^{\prime} U_{o}^{2}}{v}, y=\frac{y^{\prime} U_{o}^{2}}{v},(u, v, w)=\frac{\left(u^{\prime}, v^{\prime}, w^{\prime}\right)}{U_{o}}, \theta=\frac{\left(T^{\prime}-T_{\infty}^{\prime}\right)}{\left(T_{w}^{\prime}-T_{\infty}^{\prime}\right)}, C=\frac{\left(C^{\prime}-C_{\infty}^{\prime}\right)}{\left(C_{w}^{\prime}-C_{\infty}^{\prime}\right)}, \\
& M=\frac{\sigma \mu_{e}^{2} H_{0}^{2} v}{\rho U_{o}^{2}}, \operatorname{Pr}=\frac{\mu c_{p}}{\kappa}, S c=\frac{v}{D}, k_{r}=\frac{K_{r}^{\prime} v}{U_{0}^{2}}, G r=\frac{v g \beta\left(T_{w}^{\prime}-T_{\infty}^{\prime}\right)}{U_{o}^{3}}, \\
& G c=\frac{v g \beta^{*}\left(C_{w}^{\prime}-C_{\infty}^{\prime}\right)}{U_{o}^{3}}, E c=\frac{U_{0}^{2}}{c_{p}\left(T_{w}^{\prime}-T_{\infty}^{\prime}\right)}, R=\frac{\kappa k^{*}}{4 \sigma T_{h}^{\prime 3}}
\end{aligned}
$$

The governing equations can be obtained in the dimensionless form as:

$$
\begin{aligned}
& \frac{\partial v}{\partial y}=0 \\
& \frac{\partial u}{\partial t}+v \frac{\partial u}{\partial y}=\frac{\partial^{2} u}{\partial y^{2}}-\frac{M}{\left(1+m^{2}\right)}(u+m w)+(G r) \theta+(G c) C \\
& \frac{\partial w}{\partial t}+v \frac{\partial w}{\partial y}=\frac{\partial^{2} w}{\partial y^{2}}-\frac{M}{\left(1+m^{2}\right)}(w-m u) \\
& \frac{\partial \theta}{\partial t}+v \frac{\partial \theta}{\partial y}=\frac{1}{\operatorname{Pr}}\left(1+\frac{4}{3 R}\right) \frac{\partial^{2} \theta}{\partial y^{2}}+E c\left(\frac{\partial u}{\partial y}\right)^{2}
\end{aligned}
$$


$\frac{\partial C}{\partial t}+v \frac{\partial C}{\partial y}=\frac{1}{S c} \frac{\partial^{2} C}{\partial y^{2}}-k_{r} C$

The initial and boundary conditions (8) in the non - dimensional form are:

$t \leq 0: \quad u=0, w=0, \theta=0, C=0 \quad$ for all $y$
$t>0:\left\{\begin{array}{ll}u=0, w=0, \theta=1, C=1 & \text { at } y=0 \\ u=0, w=0, \theta=0, C=0 & \text { as } y \rightarrow \infty\end{array}\right\}$

(19)From

equation (14), we see that $v$ is either constant or a function of time $t$. Similarly solutions of equations (15) -

(18) with the boundary conditions (19) exisists only if we take

$v=\lambda t^{\frac{-1}{2}}(20)$

Forsuction $\lambda>0$ and for blowing $\lambda<0$. From (20), it can be observed that the assumption is valid only for small values of time variable.

The skin - friction due to primary velocity at the wall along $x^{\prime}-$ axis is given by $\tau_{1}=\left(\frac{\partial u}{\partial y}\right)_{y=0}$

The skin - friction due to secondary velocity at the wall along $z^{\prime}-$ axis is given by $\tau_{2}=\left(\frac{\partial w}{\partial y}\right)_{y=0}$

Rate of heat transfer (Nusselt number) due to temperature profiles is given by

$N u=\left(\frac{\partial \theta}{\partial y}\right)_{y=0}$

And rate of mass transfer (Sherwood number) due to concentration profiles is given by

$S h=\left(\frac{\partial C}{\partial y}\right)_{y=0}$

\section{Method of solution:}

By applying Galerkin finite element method for equation (15) over the element $(e),\left(y_{j} \leq y \leq y_{k}\right)$ is:

$\int_{y_{j}}^{y_{k}}\left\{N^{(e)^{T}}\left[\frac{\partial^{2} u^{(e)}}{\partial y^{2}}-\frac{\partial u^{(e)}}{\partial t}-v \frac{\partial u^{(e)}}{\partial y}-A u^{(e)}+P\right]\right\} d y=0$

Where $A=\frac{M}{1+m^{2}}, P=(G r) \theta_{i}^{j}+(G c) C_{i}^{j}-A m w_{i}^{j}$

Integrating the first term in equation (25) by parts one obtains

$N^{(e)^{T}}\left\{\frac{\partial u^{(e)}}{\partial y}\right\}_{y_{j}}^{y_{k}}-\int_{y_{j}}^{y_{k}}\left\{\frac{\partial N^{(e)^{T}}}{\partial y} \frac{\partial u^{(e)}}{\partial y}+N^{(e)^{T}}\left(\frac{\partial u^{(e)}}{\partial t}+v \frac{\partial u^{(e)}}{\partial y}+A u^{(e)}-P\right)\right\} d y=0$

Neglecting the first term in equation (26), one gets:

$\int_{y_{j}}^{y_{k}}\left\{\frac{\partial N^{(e)^{T}}}{\partial y} \frac{\partial u^{(e)}}{\partial y}+N^{(e)^{T}}\left(\frac{\partial u^{(e)}}{\partial t}+v \frac{\partial u^{(e)}}{\partial y}+A u^{(e)}-P\right)\right\} d y=0$

Let $u^{(e)}=N^{(e)} \phi^{(e)}$ be the linear piecewise approximation solution over the element $(e)\left(y_{j} \leq y \leq y_{k}\right)$, where $N^{(e)}=\left[\begin{array}{ll}N_{j} & N_{k}\end{array}\right], \phi^{(e)}=\left[\begin{array}{ll}u_{j} & u_{k}\end{array}\right]^{T}$ and $N_{j}=\frac{y_{k}-y}{y_{k}-y_{j}}, N_{k}=\frac{y-y_{j}}{y_{k}-y_{j}}$ are the basis functions.

One obtains: 


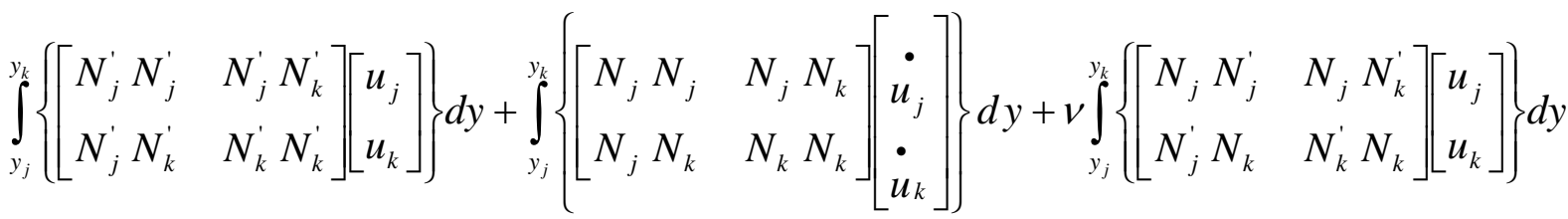

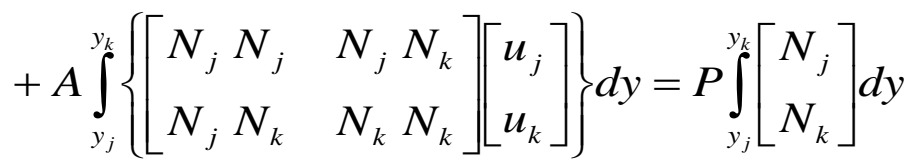

Simplifying we get

$$
\frac{1}{l^{(e)^{2}}}\left[\begin{array}{rr}
1 & -1 \\
-1 & 1
\end{array}\right]\left[\begin{array}{l}
u_{j} \\
u_{k}
\end{array}\right]+\frac{1}{6}\left[\begin{array}{ll}
2 & 1 \\
1 & 2
\end{array}\right]\left[\begin{array}{l}
\dot{u}_{j} \\
\dot{u}_{k}
\end{array}\right]+\frac{v}{2 l^{(e)}}\left[\begin{array}{ll}
-1 & 1 \\
-1 & 1
\end{array}\right]\left[\begin{array}{l}
u_{j} \\
u_{k}
\end{array}\right]+\frac{A}{6}\left[\begin{array}{ll}
2 & 1 \\
1 & 2
\end{array}\right]\left[\begin{array}{l}
u_{j} \\
u_{k}
\end{array}\right]=\frac{P}{2}\left[\begin{array}{l}
1 \\
1
\end{array}\right]
$$

Using the above equation, in order to get the difference equation at the nodes $\left(y_{i-1} \leq y \leq y_{i}\right)$ and $\left(y_{i} \leq y \leq y_{i+1}\right)$. We write the element equation for the element $\left(y_{i-1} \leq y \leq y_{i}\right)$ as $\frac{1}{l^{(e)^{2}}}\left[\begin{array}{rr}1 & -1 \\ -1 & 1\end{array}\right]\left[\begin{array}{l}u_{i-1} \\ u_{i}\end{array}\right]+\frac{1}{6}\left[\begin{array}{ll}2 & 1 \\ 1 & 2\end{array}\right]\left[\begin{array}{l}\dot{u}_{i-1} \\ \dot{u}_{i}\end{array}\right]+\frac{v}{2 l^{(e)}}\left[\begin{array}{ll}-1 & 1 \\ -1 & 1\end{array}\right]\left[\begin{array}{l}u_{i-1} \\ u_{i}\end{array}\right]+\frac{A}{6}\left[\begin{array}{ll}2 & 1 \\ 1 & 2\end{array}\right]\left[\begin{array}{l}u_{i-1} \\ u_{i}\end{array}\right]=\frac{P}{2}\left[\begin{array}{l}1 \\ 1\end{array}\right]$ and for the second element $\left(y_{i} \leq y \leq y_{i+1}\right)$ we get the element equation as

$$
\frac{1}{l^{(e)^{2}}}\left[\begin{array}{rr}
1 & -1 \\
-1 & 1
\end{array}\right]\left[\begin{array}{l}
u_{i} \\
u_{i+1}
\end{array}\right]+\frac{1}{6}\left[\begin{array}{ll}
2 & 1 \\
1 & 2
\end{array}\right]\left[\begin{array}{l}
\dot{u}_{i} \\
\dot{u}_{i+1}
\end{array}\right]+\frac{v}{2 l^{(e)}}\left[\begin{array}{ll}
-1 & 1 \\
-1 & 1
\end{array}\right]\left[\begin{array}{l}
u_{i} \\
u_{i+1}
\end{array}\right]+\frac{A}{6}\left[\begin{array}{ll}
2 & 1 \\
1 & 2
\end{array}\right]\left[\begin{array}{l}
u_{i} \\
u_{i+1}
\end{array}\right]=\frac{P}{2}\left[\begin{array}{l}
1 \\
1
\end{array}\right]
$$

where prime and dot denotes differentiation with respect to $y$ and time $t$ respectively. Assembling the above two element equations for two consecutive elements $\left(y_{i-1} \leq y \leq y_{i}\right)$ and $\left(y_{i} \leq y \leq y_{i+1}\right)$ following is obtained:

$$
\begin{aligned}
& \frac{1}{l^{(e)^{2}}}\left[\begin{array}{rrr}
1 & -1 & 0 \\
-1 & 2 & -1 \\
0 & -1 & 1
\end{array}\right]\left[\begin{array}{l}
u_{i-1} \\
u_{i} \\
u_{i+1}
\end{array}\right]+\frac{1}{6}\left[\begin{array}{ccc}
2 & 1 & 0 \\
1 & 4 & 1 \\
0 & 1 & 2
\end{array}\right]\left[\begin{array}{l}
\dot{u}_{i-1} \\
\dot{u}_{i} \\
\dot{u}_{i+1}
\end{array}\right]+\frac{v}{2 l^{(e)}}\left[\begin{array}{rrr}
-1 & 1 & 0 \\
-1 & 0 & 1 \\
0 & -1 & 1
\end{array}\right]\left[\begin{array}{l}
u_{i-1} \\
u_{i} \\
u_{i+1}
\end{array}\right] \\
& +\frac{A}{6}\left[\begin{array}{rrr}
2 & 1 & 0 \\
1 & 4 & 1 \\
0 & 1 & 2
\end{array}\right]\left[\begin{array}{l}
u_{i-1} \\
u_{i} \\
u_{i+1}
\end{array}\right]=\frac{P}{2}\left[\begin{array}{l}
1 \\
2 \\
1
\end{array}\right]
\end{aligned}
$$

Now put row corresponding to the node $i$ to zero, from equation (27) the difference schemes with $l^{(e)}=h$ is:

$$
\frac{1}{h^{2}}\left[-u_{i-1}+2 u_{i}-u_{i+1}\right]+\frac{1}{6}\left[\dot{u}_{i-1}+4 \dot{u}_{i}+\dot{u}_{i+1}\right]+\frac{v}{2 h}\left[-u_{i-1}+u_{i+1}\right]+\frac{A}{6}\left[u_{i-1}+4 u_{i}+u_{i+1}\right]=P
$$

Applying the trapezoidal rule, following system of equations in Crank - Nicholson method are obtained:

$A_{1} u_{i-1}^{n+1}+A_{2} u_{i}^{n+1}+A_{3} u_{i+1}^{n+1}=A_{4} u_{i-1}^{n}+A_{5} u_{i}^{n}+A_{6} u_{i+1}^{n}+P^{*}(29)$

Now from equations (16), (17) and (18), following equations are obtained:

$$
B_{1} w_{i-1}^{n+1}+B_{2} w_{i}^{n+1}+B_{3} w_{i+1}^{n+1}=B_{4} w_{i-1}^{n}+B_{5} w_{i}^{n}+B_{6} w_{i+1}^{n}+Q^{*}
$$




$$
\begin{aligned}
& G_{1} \theta_{i-1}^{n+1}+G_{2} \theta_{i}^{n+1}+G_{3} \theta_{i+1}^{n+1}=G_{4} \theta_{i-1}^{n}+G_{5} \theta_{i}^{n}+G_{6} \theta_{i+1}^{n}+R^{*} \\
& J_{1} C_{i-1}^{n+1}+J_{2} C_{i}^{n+1}+J_{3} C_{i+1}^{n+1}=J_{4} C_{i-1}^{n}+J_{5} C_{i}^{n}+J_{6} C_{i+1}^{n}
\end{aligned}
$$

where $A_{1}=2+A k-3 v r h-6 r, A_{2}=4 A r h+12 r+8, A_{3}=2+A k+3 v r h-6 r$

$$
\begin{aligned}
& A_{4}=2-A k+3 v r h-6 r, A_{5}=8-4 A r h-12 r, A_{6}=2-A k-3 v r h+6 r \\
& B_{1}=2+A k-3 v r h-6 r, B_{2}=4 A r h+12 r+8, B_{3}=2+A k+3 v r h-6 r \\
& B_{4}=2-A k+3 v r h-6 r, B_{5}=8-4 A r h-12 r, B_{6}=2-A k-3 v r h+6 r \\
& G_{1}=2(\operatorname{Pr})-3 v(\operatorname{Pr}) r h-6 Z r, G_{2}=8(\operatorname{Pr})+12 Z r, G_{3}=2(\operatorname{Pr})+3 v(\operatorname{Pr}) r h-6 Z r, \\
& G_{4}=2(\operatorname{Pr})+3 v(\operatorname{Pr}) r h+6 Z r, G_{5}=8(\operatorname{Pr})-12 Z r, G_{6}=2(\operatorname{Pr})-3 v(\operatorname{Pr}) r h+6 Z r, \\
& J_{1}=2(S c)-3 v(S c) r h-6 r+\left(k_{r}\right)(S c) k, J_{2}=8(S c)+12 r+4\left(k_{r}\right)(S c) r h, \\
& J_{3}=2(S c)+3 v(S c) r h-6 r+\left(k_{r}\right)(S c) k, J_{4}=2(S c)+3 v(S c) r h+6 r-\left(k_{r}\right)(S c) k, \\
& J_{5}=8(S c)-12 r-4\left(k_{r}\right)(S c) r h, J_{6}=2(S c)-3 v(S c) r h+6 r-\left(k_{r}\right)(S c) k \\
& P^{*}=12 h P k=12 h k(G r) \theta_{i}^{j}+12 h k(G c) C_{i}^{j}-12 h k A m w_{i}^{j}, Q^{*}=12 Q h k=12 h k A m u_{i}^{j}, \\
& Y^{*}=12 k Y=12 k(\operatorname{Pr})(E c)\left(\frac{\partial u_{i}^{j}}{\partial y}\right)^{2}, Z=1+\frac{4}{3 R}
\end{aligned}
$$

Here $r=\frac{k}{h^{2}}$ and $h, k$ are mesh sizes along $y-$ direction and $t-$ direction respectively. Index $i$ refers to space and $j$ refers to the time. In the equations (29), (30), (31) and (32) taking $i=1(1) n$ and using boundary conditions (19), then the following system of equations are obtained:

$$
A_{i} X_{i}=B_{i} \quad i=1(1) 4
$$

Where $A_{i}^{\prime}$ s are matrices of order $n$ and $X_{i}, B_{i}^{\prime}$ s are column matrices having $n$ - components. The solutions of above system of equations are obtained by using Thomas algorithm for primary velocity, secondary velocity, temperature and concentration. Also, numerical solutions for these equations are obtained by $C-$ programme. In order to provethe convergence and stability of Galerkin finite element method, the same $C$ programme was runwith smaller values of $h$ and $k$ and no significant change was observed in the values of $u$, $w, \theta$ and $C$. Hence the Galerkinfinite element method is stable and convergent.

\section{Results and Discussions:}

The problem ofeffect of Hall current on an unsteady magnetohydrodynamic flow of an electrically conducting incompressible fluid along an infinite vertical porous plate with viscous dissipation has been studiedand solved by using Galerkin finite element method. The effects of material parameters such as thermal Grashof number $(G r)$, solutal Grashof number $(G c)$ Prandtl number $(\operatorname{Pr})$, Schmidt number $(S c)$, Hartmann number $(M)$, Hall parameter $(m)$, Eckertnumber $(E c)$, Thermal radiation parameter $(R)$, Chemical reaction parameter $\left(k_{r}\right)$ and Transpiration cooling parameter $(\lambda)$ separately in order to clearly observe their respective effects on the primary velocity, secondary velocity, temperature and concentration profiles of the flow. And also numerical values of skin - friction coefficients $\left(\tau_{1} \& \tau_{2}\right)$, Nusselt number $(\mathrm{Nu})$ and Sherwood number $(S h)$ have been discussed in table 1, 2 and 3 respectively. During the course of numerical calculations of the primary velocity $(u)$, secondary velocity $(w)$, temperature $(\theta)$ and concentration $(C)$ the values of the Prandtl number are chosen for Mercury $(\operatorname{Pr}=0.025)$, Air at $25^{\circ} C$ and one atmospheric pressure $(\operatorname{Pr}=0.71)$, Water $(\operatorname{Pr}=7.00)$ and Water at $4^{\circ} C(\operatorname{Pr}=11.62)$.To focus out attention on numerical values of the results obtained in the study, the values of $S c$ are chosen for the gases representing diffusing chemical species of most common interest in air namely Hydrogen( $S c=0.22)$, Helium $(S c=0.30)$, Water $-\operatorname{vapour}(S c=0.60)$, Oxygen ( $S c=0.66$ ) and Ammonia ( $S c=0.78$ ). For the physical significance, the numerical discussions in the problem and at $t=1.0$, stable values for primary velocity, secondary velocity, temperature and concentration fields are 
obtained. To examine the effect of parameters related to the problem on the velocity field and skin - friction numerical computations are carried out at $\operatorname{Pr}=0.71$. To find out the solution of this problem, we have placed an infinite vertical plate in a finite length in the flow. Hence, we solve the entire problem in a finite boundary. However, in the graphs, the $y$ values vary from 0 to 4 , and the velocity, temperature, and concentration tend to zero as $y$ tends to 4 . This is true for any value of $y$. Thus, we have considered finite length.

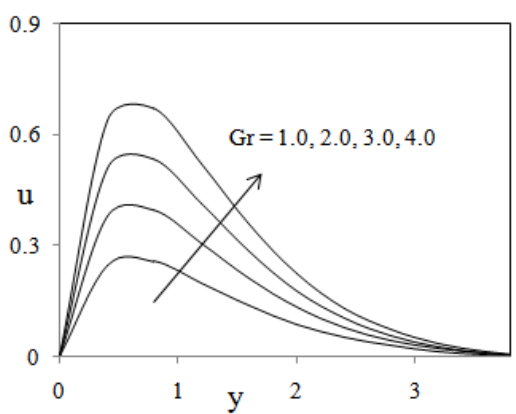

Figure 2. Effect of thermal Grashof number $G r$ onprimary velocity profiles $u$

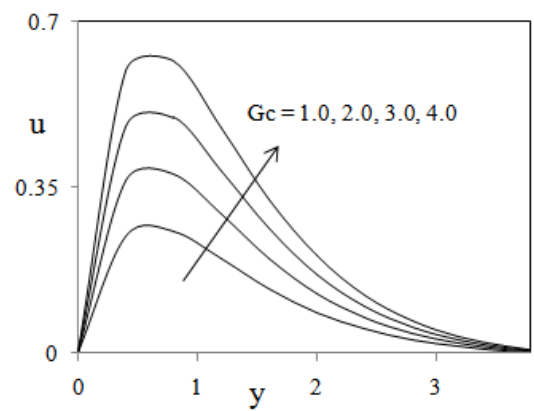

Figure 3. Effect of solutal Gralkhof number $G c$ on primary velocity profiles $u$

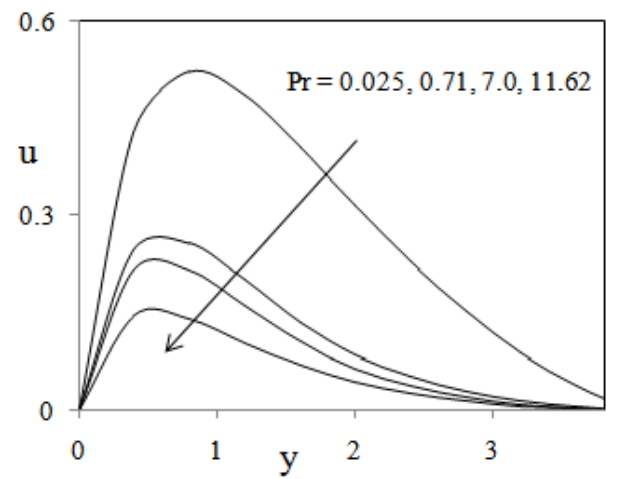

Figure 4. Effect of Brandt number Pr onprimary velocity profiles $u$

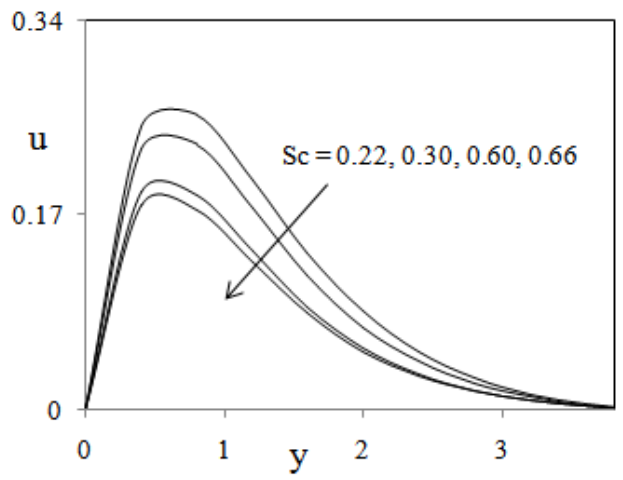

Figure 5. Effect of Schmidt number SC onprimary velocity profiles $u$ 
The temperature and the species concentration are coupled to the velocity via Grashof number $(G r)$ and Modified Grashof number $(G c)$ as seen in equation (15).For various values of Grashof number and Modified Grashof number, the velocity profiles $u$ are plotted in figures (2) and (3). The Grashof number $(G r)$ signifies the relative effect of the thermal buoyancy force to the viscous hydrodynamic force in the boundary layer. As expected, it is observed that there is a rise in the velocity due to the enhancement of thermal buoyancy force. Also, as $G r$ increases, the peak values of the velocity increases rapidly near the porous plate and then decays smoothly to the free stream velocity. The Modified Grashof number $(G c)$ defines the ratio of the species buoyancy force to the viscous hydrodynamic force. As expected, the fluid velocity increases and the peak value is more distinctive due to increase in the species buoyancy force. The velocity distribution attains a distinctive maximum value in the vicinity of the plate and then decreases properly to approach the free stream value. It is noticed that the velocity increases with increasing values of Modified Grashof number $(G c)$.

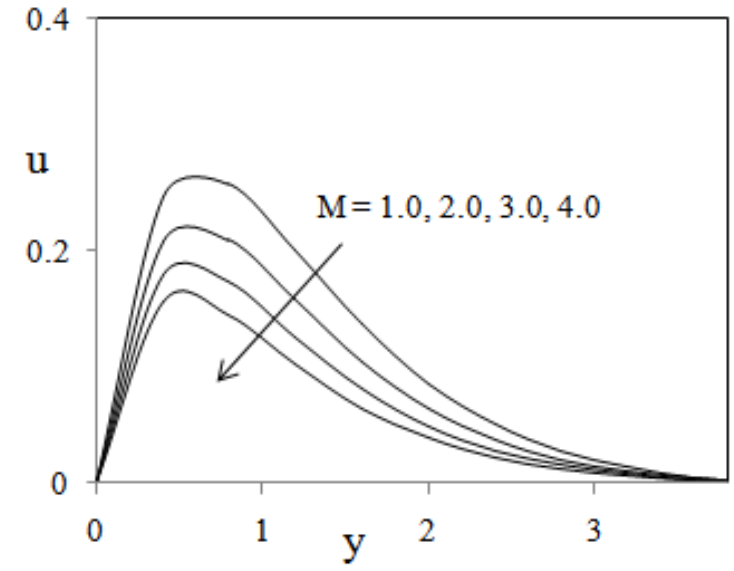

Figure 6. Effect of Hartmann number $M$ on primary velocity profiles $u$

Figure (4) depicts the effect of Prandtl number on primary velocity profiles in presence of foreign species such as Mercury $(\operatorname{Pr}=0.025)$, Air at $25^{\circ} \mathrm{C}$ and one atmospheric pressure

$(\mathrm{Pr}=0.71)$, Water $(\operatorname{Pr}=7.00)$ and Water at $4^{\circ} C(\operatorname{Pr}=11.62)$ are shown in figure (4). We observe that from figure (4), the primary velocity decreases with increasing of Prandtl number $(\operatorname{Pr})$. The nature of primary velocity profiles in presence of foreign species such as Hydrogen $(S c=0.22)$, Oxygen $(S c=0.60)$, Water - vapour $(S c=0.66$ ) and Ammonia $(S c=0.78)$ are shown in figure (5).

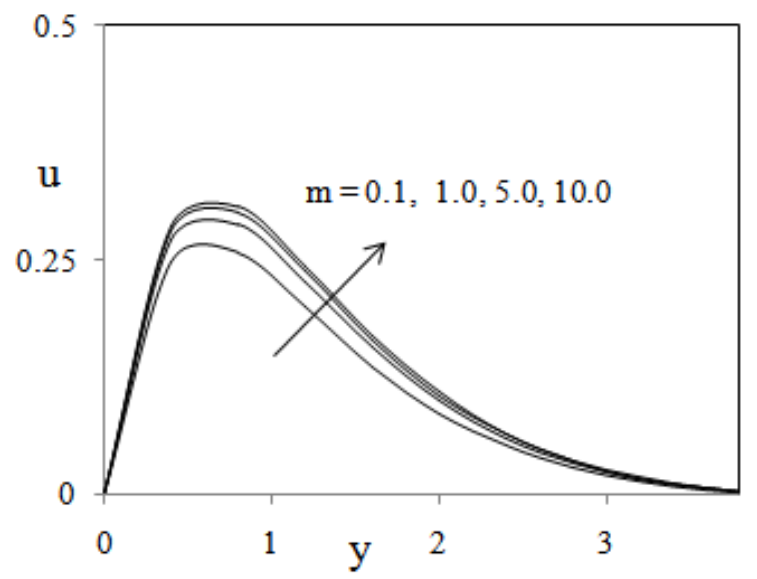

Figure 7. Effect of Hall parameter $m$ on primary velocity profiles $u$

The flow field suffers a decrease in primary velocity at all points in presence of heavier diffusing species.The effect of the Hartmann number $(M)$ is shown in figure (6). It is observed that, the primary velocity of the fluid decreases with the increasing of the magnetic field number values. The decrease in the primary velocity as the Hartmann number $(M)$ increases is because the presence of a magnetic field in an electrically 
conducting fluid introduces a force called the Lorentz force, which acts against the flow, if the magnetic field is applied in thenormal direction, as in the present study. This resistive force slows down the fluid velocity component as shown in figure (6). Figure (7) depicts the primary velocity profiles as the Hall parameter $m$ increases. We see that $u$ increases as $m$ increases. It can also be observed that $u$ profiles approach their classical values when Hall parameter $m$ becomes large $(m>5)$.

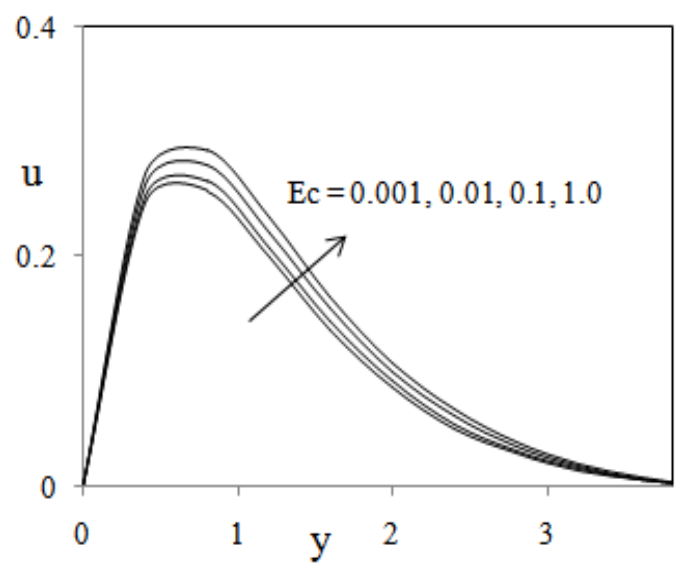

Figure 8. Effect of Eckert number $E c$ on primary velocity profiles $u$

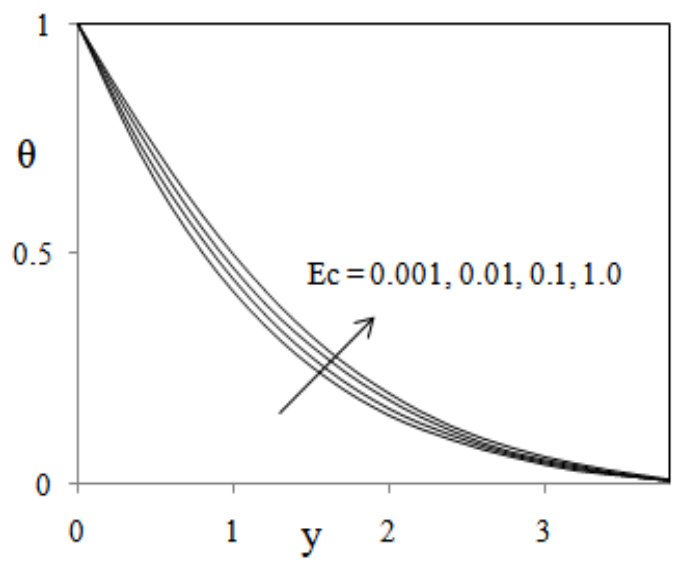

Figure 9. Effect of Eckert number $E c$ on temperature profiles $\theta$

The influence of the viscous dissipation parameter i.e., the Eckert number $(E c)$ on the velocity and temperature are shown in figures (8) and (9) respectively. The Eckert number $(E c)$ expresses the relationship between the kinetic energy in the flow and the enthalpy. It embodies the conversion of kinetic energy into internal energy by work done against the viscous fluid stresses. Greater viscous dissipative heat causes a rise in the temperature as well as the velocity. This behavior is evident from figures (8) and (9).The effect of the thermal radiation parameter $R$ on the primary velocity and temperature profiles in the boundary layer are illustrated in figures (10) and (11) respectively. Increasing the thermal radiation parameter $R$ produces significant increase in the thermal condition of the fluid and its thermal boundary layer. This increase in the fluid temperature induces more flow in the boundary layer causing the velocity of the fluid there to increase. 


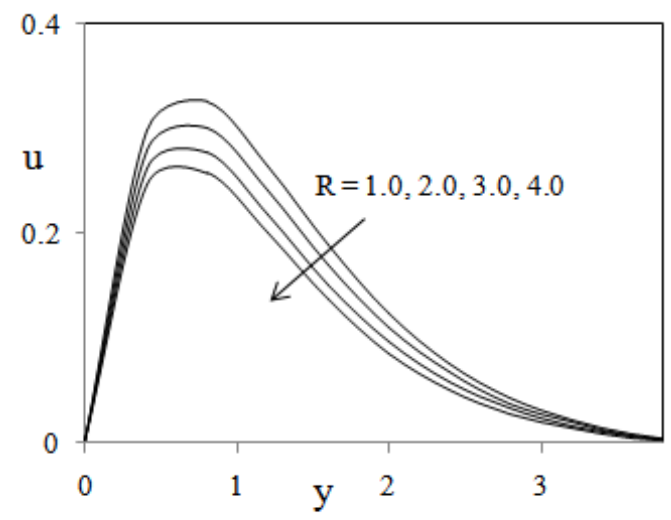

Figure 10. Effect of Themal radiation parameter $R$ on primary velocity profiles $u$

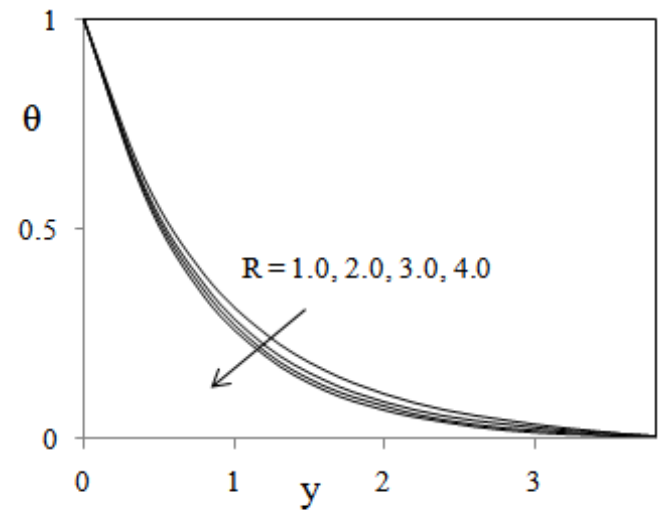

Figure 11. Effect of Themal radiation parameter $R$ on temperature profiles $\theta$

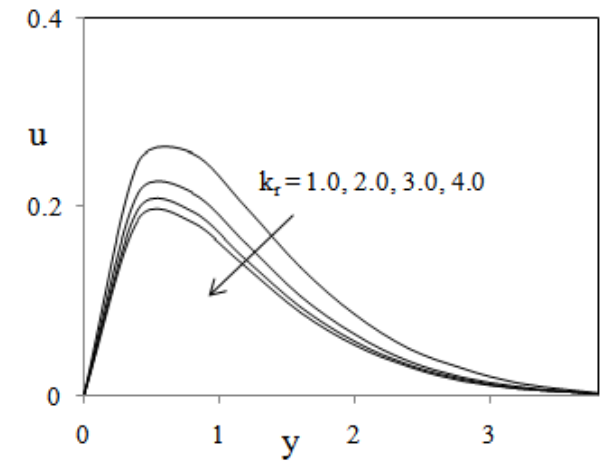

Figure 12. Effect of Chemical reaction parameter $k_{r}$ onPrimary velocity profiles $u$

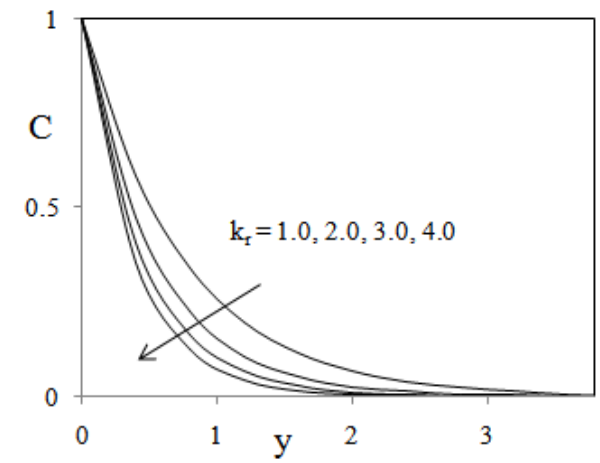

Figure 13. Effect of Chemical reaction parameter $k_{r}$ on concentration profiles $C$ 
Figures (12) and (13) display the effects of the chemical reaction parameter $\left(k_{r}\right)$ on the velocity and concentration profiles, respectively. As expected, the presence of the chemical reaction significantly affects the concentration profiles as well as the velocity profiles. It should be mentioned that the studied case is for a destructive chemical reaction $\left(k_{r}\right)$. In fact, as chemical reaction $\left(k_{r}\right)$ increases, the considerable reduction in the velocity profiles is predicted, and the presence of the peak indicates that the maximum value of the velocity occurs in the body of the fluid close to the surface but not at the surface. Also, with an increase in the chemical reaction parameter, the concentration decreases. It is evident that the increase in the chemical reaction $\left(k_{r}\right)$ significantly alters the concentration boundary layer thickness but does not alter the momentum boundary layers.

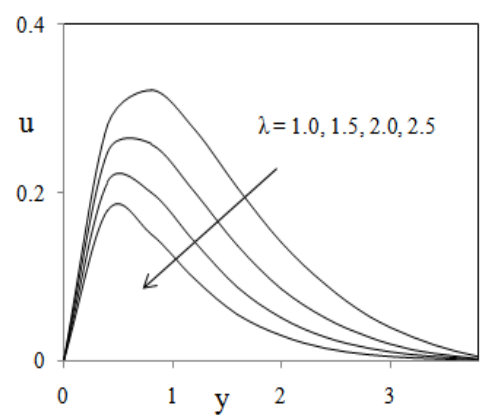

Figure 14. Effect of Transpiration cooling parameter $\lambda$ on primary velocity profiles $u$

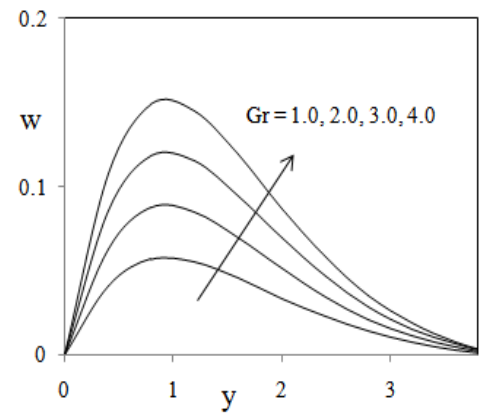

Figure 15. Effect of thermal Grashof number $G r$ on secondary velocity profiles $w$

Fromfigure (14) shows that the primary velocity profiles against $y$ for several values of the transpiration cooling parameter $(\lambda)$ keeping other parameters of the flow field are constant. The transpiration cooling parameter is found to retard the primary velocity of the flow field at all points. The reduction in primary velocity at any point of the flow field is faster as the transpiration cooling parameter becomes larger. One interesting inference of this finding is greater transpiration cooling leads to a faster reduction in the primary velocity of the flow field.

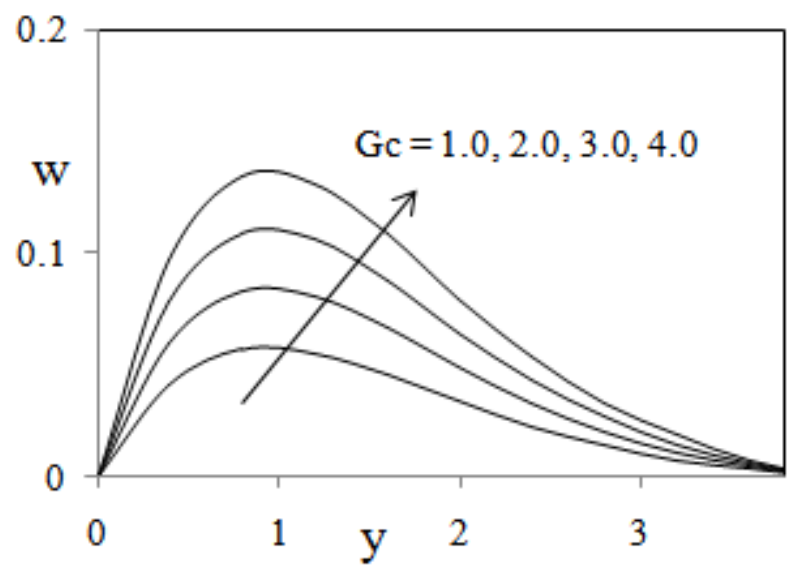

Figure 16. Effect of solutal Grashof number $G c$ on secondary velocity profiles $w$ 


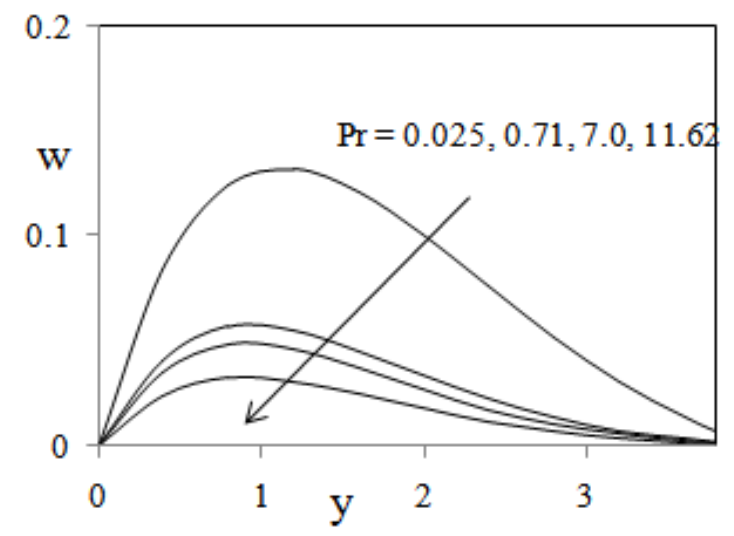

Figure 17. Effect of Prandtl number Pr on secondary velocity profiles $w$

In figures (15) and (16), we see the influence of the both heat and mass transfer on secondary velocity of the flow. It can be seen that as both the heat and mass transfer increases, this secondary velocity component increases as well. Figure (17) depicts the effect of Prandtl number on secondary velocity profiles in presence of foreign species such as Mercury $(\operatorname{Pr}=0.025)$, Air at $25^{\circ} C$ and one atmospheric pressure $(\operatorname{Pr}=0.71)$, Water ( $\mathrm{Pr}=7.00)$ and Water at $4^{\circ} \mathrm{C}(\mathrm{Pr}=11.62)$. We observe that from figure (17) the velocity is decreasing with increasing of Prandtl number $(\operatorname{Pr})$. The effect of Schmidt number $(S c)$ on secondary velocity is as shown in the figure (18). The nature of primary velocity profiles in presence of foreign species such as Hydrogen ( $S c=$ $0.22)$, Oxygen $(S c=0.60)$, Water - vapour $(S c=0.66)$ and Ammonia $(S c=0.78)$. We observe that from figure (18) the velocity is decreasing with increasing of Schmidt number $(S c$ ). In figure (19) we have the influence of the Hartmann number $(M)$ on the secondary velocity. It can be seen that as the values of this parameter increases, the secondary velocity increases. In figure (20), we see that $w$ profiles increase for $m<1$ and decrease for $m>1$. The effects of Eckertnumber $(E c)$ and Thermal radiation parameter $(R)$ on secondary velocity are shown in the figures (21) and (22) respectively. From these figures we observe that the secondary velocity increases with increasing values of Eckertnumber $(E c)$ and decreasing with increasing values of Thermal radiation parameter $(R)$. In figure (23), we have the effect of the chemical reaction parameter $\left(k_{r}\right)$ on the secondary velocity. It can be seen that as the values of this parameter increases, the secondary velocity also increases.Figure (24) shows that the secondary velocity profiles against $y$ for different values of the transpiration cooling parameter $(\lambda)$. As the transpiration cooling parameter is to retard the secondary velocity.

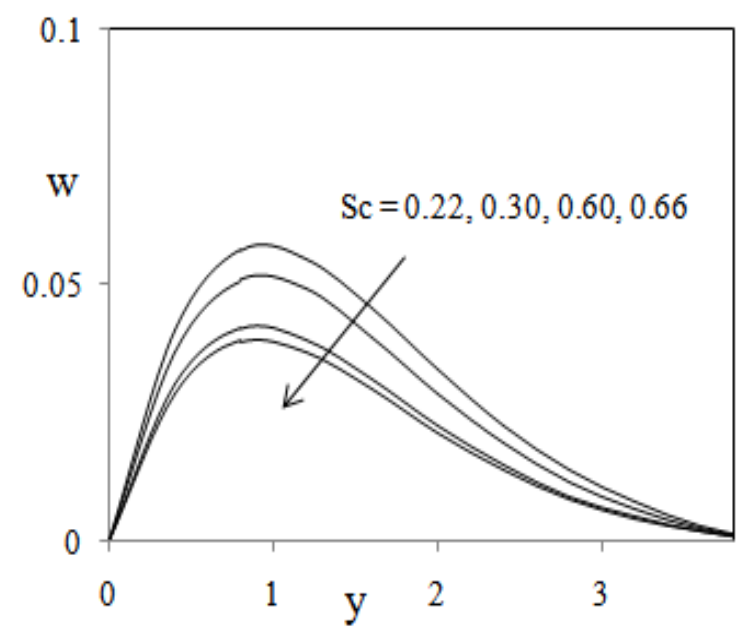

Figure 18. Effect of Schmidt number $S c$ on secondary velocity profiles $w$ 


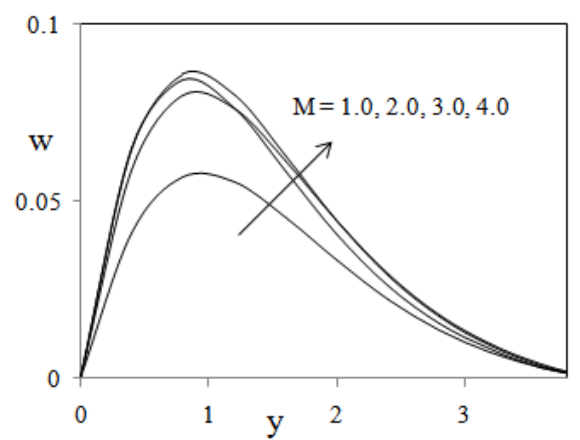

Figure 19. Effect of Hartmann number $M$ on secondary velocity profiles $w$

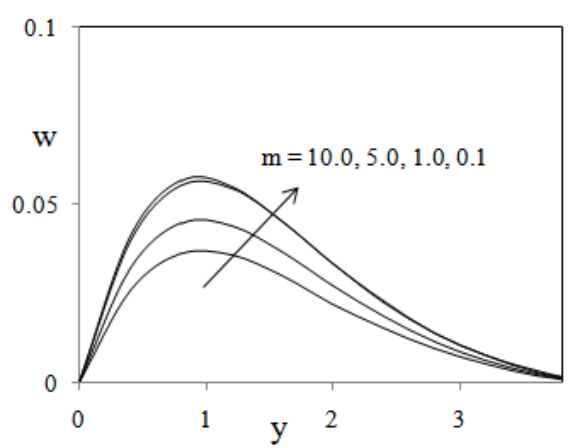

Figure 20. Effect of Hall parameter $m$ on secondary velocity profiles $w$

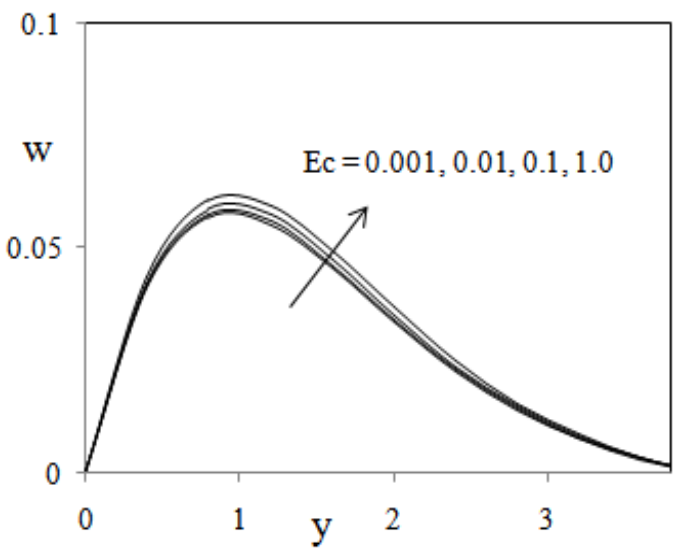

Figure 21. Effect of Eckert number $E c$ on Secondary velocity profiles $w$

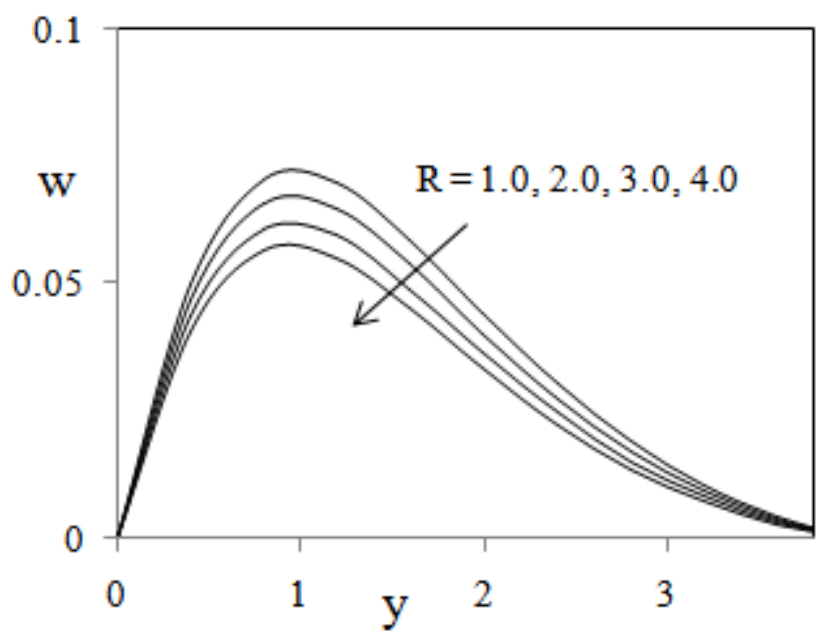

Figure 22. Effect of Thermal radiation parameter $R$ on Secondary velocity profiles $w$ 


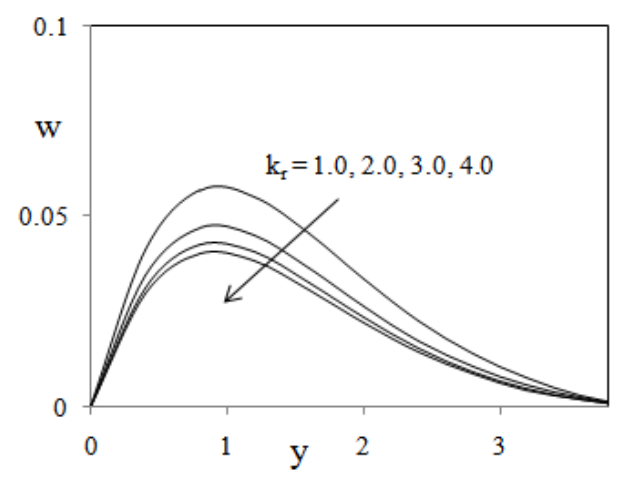

Figure 23. Effect of Chemical reaction parameter $k_{r}$ on Secondary velocity profiles $w$

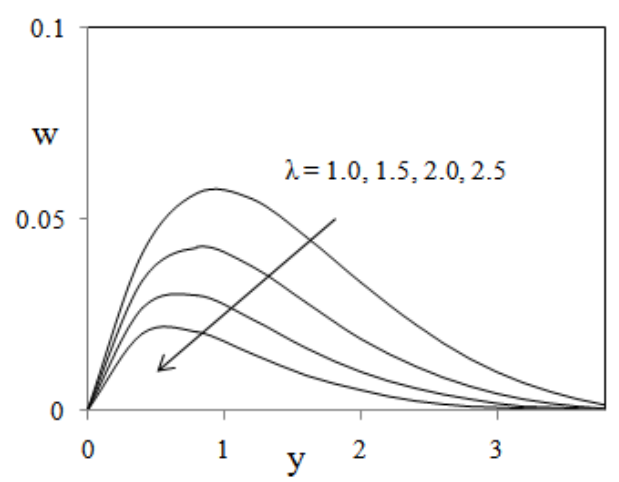

Figure 24. Effect of Transpiration cooling parameter $\lambda$ on secondary velocity profiles $w$

In figure (25) we depict the effect of Prandtl number( $\operatorname{Pr}$ ) on the temperature field. It is observed that an increase in the Prandtl number leads to decrease in the temperature field. Also, temperature field falls more rapidly for water in comparison to air and the temperature curve is exactly linear for mercury, which is more sensible towards change in temperature. From this observation it is conclude that mercury is most effective for maintaining temperature differences and can be used efficiently in the laboratory. Air can replace mercury, the effectiveness of maintaining temperature changes are much less than mercury. However, air can be better and cheap replacement for industrial purpose.This is because, either increase of kinematic viscosity or decrease of thermal conductivity leads to increase in the value of Prandtl number( $\operatorname{Pr})$. Hence temperature decreases with increasing of Prandtl number ( Pr ).

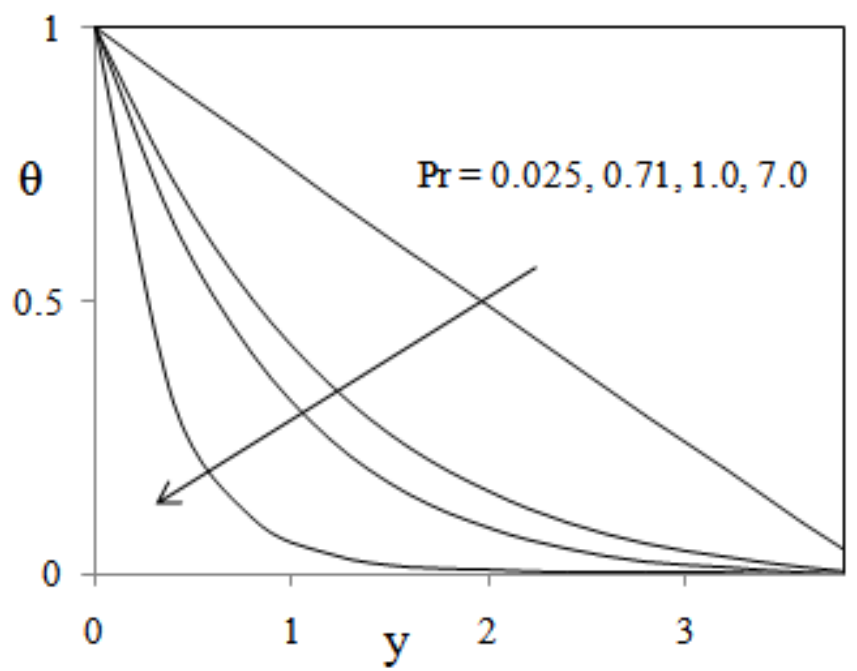

Figure 25. Effect of Prandtl number Pr on temperature profiles $\theta$ 


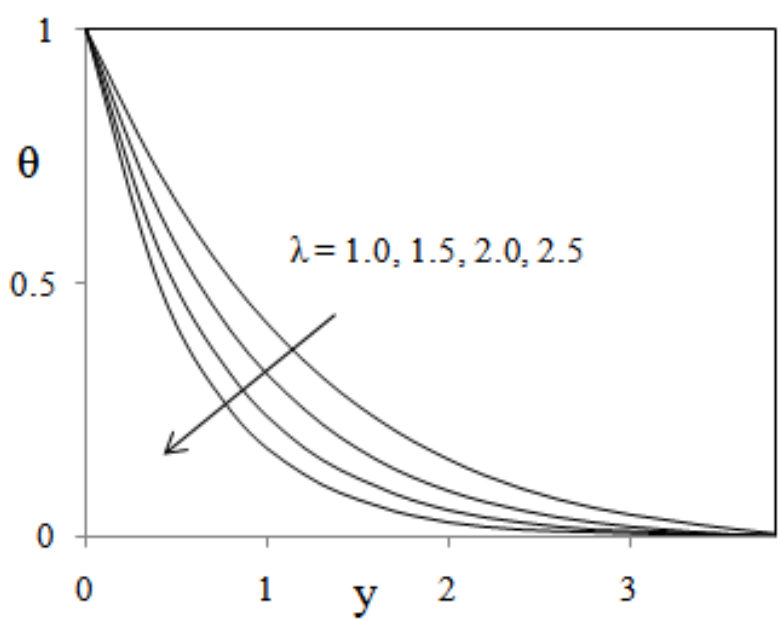

Figure 26. Effect of Transpiration cooling parameter $\lambda$ on temperature profiles $\theta$

From figure (26) depicts the temperature profiles against $y$ for various values of transpiration cooling parameter $(\lambda)$ keeping other parameters are constant. Transpiration cooling parameter is found to decrease the temperature of the flow field at all points. The effects of Schmidt number $(S c)$ and Transpiration cooling parameter $(\lambda)$ on the concentration field are presented in figures (27) and (28).

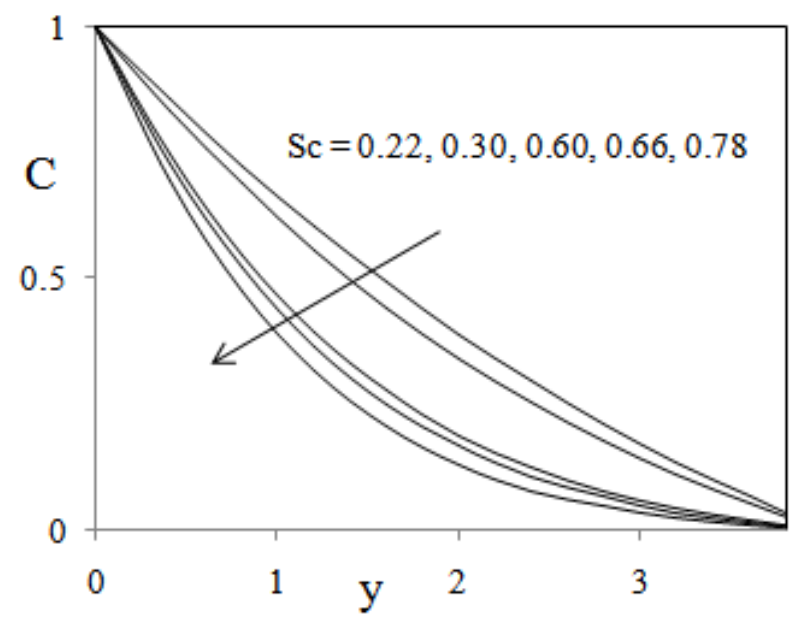

Figure 27. Effect of Schmidt number $S c$ on concentration profiles $C$

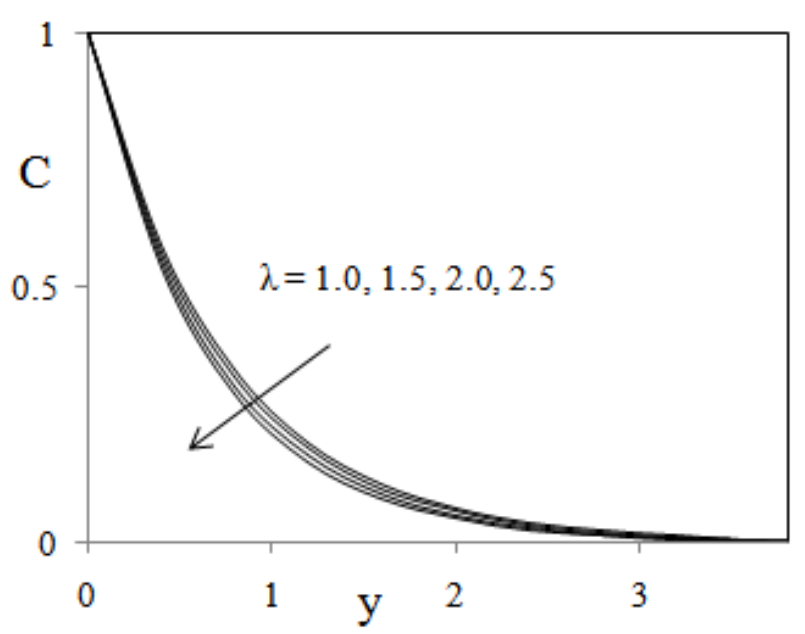

Figure 28. Effect of Transpiration cooling parameter $\lambda$ on concentration profiles $C$ 
Figure (27) shows the concentration field due to variation in Schmidt number (Sc) for the gasses Hydrogen, Helium,Water - vapour, Oxygen and Ammonia. It is observed that concentration field is steadily for Hydrogen and falls rapidly for Oxygen and Ammonia in comparison to Water - vapour. Thus Hydrogen can be used for maintaining effective concentration field and Water - vapour can be used for maintaining normal concentration field. Figure (28) shows the plot of concentration distribution against $y$ for different values of transpiration cooling parameter $(\lambda)$ and fixed $S c=0.22$. A comparative study of the curves of the above figure shows that the concentration distribution of the flow field decreases faster as the transpiration cooling parameter $(\lambda)$ becomes larger. Thus greater transpiration cooling leads to a faster decrease in concentration of the flow field.

Table - (1) shows the variation of different values $G r, G c$, Pr, Sc, M, $m, E c, R, k_{r}$ and $\lambda$ on shearing stress $\tau_{1}$ and $\tau_{2}$. From this table it is concluded that the magnitude of shearing stress $\tau_{1}$ and $\tau_{2}$ increase as the values of $G r, G c, m, E c$ increaseand this behavior is found just reverse with the increase of $\operatorname{Pr}, S c, M, R, \lambda$ and $k_{r}$. Table - (2) shows the variation of Nusselt number $(N u)$ different values $\operatorname{Pr}, R$, $E c$ and $\lambda$. From this table it is concluded that the Nusselt number $(N u)$ increases as the value of $E c$ increases and this behavior is found just reverse with the increase of $\operatorname{Pr}, R$, and $\lambda$.Table - (3) shows the variation of Sherwood number $(S h)$ different values $S c, k_{r}$ and $\lambda$. From this table it is concluded that Sherwood number $(S h)$ decreases with the increasing values of $S c, \lambda$ and $k_{r}$.In order to ascertain the accuracy of the numerical results, the present skin - friction $\left(\tau_{1}\right)$ results are compared with the previous skin friction ( $\tau_{1}^{*}$ ) results of Sriramulu et al. [19] intable - (4). They are found to be in an excellent agreement.

Table 1. Variation of shearing stress $\tau_{1}$ and $\tau_{2}$ for different values of $G r, G c, S c, \operatorname{Pr}, M, m, S r, D u, k_{r}$ and $\lambda$

\begin{tabular}{|c|c|c|c|c|c|c|c|c|c|c|c|}
\hline$G r$ & $G c$ & $\operatorname{Pr}$ & $S c$ & $M$ & $m$ & $R$ & $E c$ & $k_{r}$ & $\lambda$ & $\tau_{1}$ & $\tau_{2}$ \\
\hline 1.0 & 1.0 & 0.71 & 0.22 & 2.0 & 0.5 & 1.0 & 0.001 & 1.0 & 0.5 & 1.1576 & 0.2336 \\
\hline 2.0 & 1.0 & 0.71 & 0.22 & 2.0 & 0.5 & 1.0 & 0.001 & 1.0 & 0.5 & 1.1981 & 0.3404 \\
\hline 1.0 & 2.0 & 0.71 & 0.22 & 2.0 & 0.5 & 1.0 & 0.001 & 1.0 & 0.5 & 2.0593 & 0.3604 \\
\hline 1.0 & 1.0 & 7.00 & 0.22 & 2.0 & 0.5 & 1.0 & 0.001 & 1.0 & 0.5 & 0.9894 & 0.1404 \\
\hline 1.0 & 1.0 & 0.71 & 0.60 & 2.0 & 0.5 & 1.0 & 0.001 & 1.0 & 0.5 & 1.1057 & 0.2180 \\
\hline 1.0 & 1.0 & 0.71 & 0.22 & 4.0 & 0.5 & 1.0 & 0.001 & 1.0 & 0.5 & 0.9694 & 0.1908 \\
\hline 1.0 & 1.0 & 0.71 & 0.22 & 2.0 & 1.0 & 1.0 & 0.001 & 1.0 & 0.5 & 1.2419 & 0.4211 \\
\hline 1.0 & 1.0 & 0.71 & 0.22 & 2.0 & 0.5 & 2.0 & 0.001 & 1.0 & 0.5 & 1.2603 & 0.2145 \\
\hline 1.0 & 1.0 & 0.71 & 0.22 & 2.0 & 0.5 & 1.0 & 0.100 & 1.0 & 0.5 & 1.1446 & 0.2475 \\
\hline 1.0 & 1.0 & 0.71 & 0.22 & 2.0 & 0.5 & 1.0 & 0.001 & 2.0 & 0.5 & 1.0035 & 0.1956 \\
\hline 1.0 & 1.0 & 0.71 & 0.22 & 2.0 & 0.5 & 1.0 & 0.001 & 1.0 & 1.0 & 1.1159 & 0.1876 \\
\hline
\end{tabular}

Table 2. Variation of Nusselt number $(N u)$ for different values of $\operatorname{Pr}, D u$ and $\lambda$

\begin{tabular}{|c|c|c|c|c|}
\hline $\operatorname{Pr}$ & $E c$ & $R$ & $\lambda$ & $N u$ \\
\hline 0.71 & 0.001 & 1.0 & 0.5 & 1.8975 \\
\hline 7.00 & 0.001 & 1.0 & 0.5 & 0.9948 \\
\hline 0.71 & 0.100 & 1.0 & 0.5 & 2.0068 \\
\hline 0.71 & 0.001 & 2.0 & 0.5 & 1.6597 \\
\hline 0.71 & 0.001 & 1.0 & 1.0 & 1.5946 \\
\hline
\end{tabular}

Table 3. Variation of Sherwood number $(S h)$ for different values of $S c, S r, k_{r}$ and $\lambda$

\begin{tabular}{|c|c|c|c|}
\hline$S c$ & $k_{r}$ & $\lambda$ & $S h$ \\
\hline 0.22 & 1.0 & 0.5 & 1.4459 \\
\hline 0.30 & 1.0 & 0.5 & 1.1493 \\
\hline 0.22 & 2.0 & 0.5 & 1.3971 \\
\hline 0.22 & 1.0 & 1.0 & 1.2290 \\
\hline
\end{tabular}

Table 4: Comparison of present Skin - Friction results $\left(\tau_{1}\right)$ with the Skin - Friction results obtained by Sriramulu et al. [19] for different values of $G r, G c, \operatorname{Pr}, S c, M, m$ and $\lambda$ 


\begin{tabular}{|c|c|c|c|c|c|c|c|c|}
\hline$G r$ & $G c$ & $\operatorname{Pr}$ & $S c$ & $M$ & $m$ & $\lambda$ & $\tau_{1}$ & $\tau_{1}^{*}$ \\
\hline 1.0 & 1.0 & 0.71 & 0.22 & 2.0 & 0.5 & 0.5 & 1.1472 & 1.1469 \\
\hline 2.0 & 1.0 & 0.71 & 0.22 & 2.0 & 0.5 & 0.5 & 1.4361 & 1.4353 \\
\hline 1.0 & 2.0 & 0.71 & 0.22 & 2.0 & 0.5 & 0.5 & 1.6958 & 1.6941 \\
\hline 1.0 & 1.0 & 7.00 & 0.22 & 2.0 & 0.5 & 0.5 & 0.6381 & 0.6365 \\
\hline 1.0 & 1.0 & 0.71 & 0.60 & 2.0 & 0.5 & 0.5 & 1.0736 & 1.0725 \\
\hline 1.0 & 1.0 & 0.71 & 0.22 & 2.0 & 0.5 & 0.5 & 0.7694 & 0.7685 \\
\hline 1.0 & 1.0 & 0.71 & 0.22 & 4.0 & 0.5 & 0.5 & 1.2419 & 1.2410 \\
\hline 1.0 & 1.0 & 0.71 & 0.22 & 2.0 & 1.0 & 0.5 & 1.1403 & 1.1395 \\
\hline
\end{tabular}

\section{Conclusions:}

The study of effect of hall current on an unsteady magnetohydrodynamic flow of an electrically conducting incompressible fluid along a porous flat plate with thermal diffusion, diffusion thermo and chemical reaction is studied. The dimensionless equations are solved by using Galerkin finite element method. The effects of primary velocity, secondary velocity, temperature and concentration for different parameters like $G r, G c, \operatorname{Pr}$,

Sc, $M, m, E c, R, k_{r}$ and $\lambda$ are studied. The study concludes the following results.

1. It is observed that the primary velocity $(u)$ of the fluid increases with the increasing of parameters $G r$, $G c, m, E c$ and decreases with the increasing of parameters $\operatorname{Pr}, S c, R, M, k_{r}$ and $\lambda$.

2. It is observed that secondary velocity ( $w$ ) of the fluid increases with the increasing of parameters $G r$, $G c, M, m, E c$ and decreases with the increasing of parameters $\operatorname{Pr}, R, S c, k_{r}$ and $\lambda$.

3. The fluid temperature increases with the increasing of $E c$ and decreases with the increasing of $\operatorname{Pr}, R$ and $\lambda$.

4. The concentration of the fluid decreases with the increasing of $S c, k_{r}$ and $\lambda$.

5. From table (1), it is concluded that the magnitude of shearing stress $\tau_{1}$ and $\tau_{2}$ increases with the increasing values of $G r, G c, m, E c$ and this behavior is found just reverse with the increasing of $\operatorname{Pr}, S c$, $M, R, \lambda$ and $k_{r}$.

6. From table (2), it is concluded that the Nusselt number $(N u)$ increases with the increasing values of $E c$ and this behavior is found just reverse with the increasing of $R, \operatorname{Pr}$ and $\lambda$.

7. From table (3), it is concluded that the Sherwood number $(S h)$ decreases with the increasing of $S c, k_{r}$ and $\lambda$.

8. On comparing the skin - friction $\left(\tau_{1}\right)$ results with the skin - friction $\left(\tau_{1}^{*}\right)$ results of Sriramulu et al. [19] it can be seen that they agree very well.

\section{References:}

[1]. Abdul Maleque, Kh. and Abdur Sattar, Md., 2005. The Effects of Variable properties and Hall current on steady MHD laminar convective fluid flow due to a porous rotating disk. Int. Journal of Heat and Mass Transfer, 48, $4460-4466$.

[2]. Ajay Kumar Singh, 2003. MHD Free - convection and Mass Transfer Flow with Hall Current Viscous Dissipation, Joule Heating and Thermal Diffusion, Indian Journal of Pure and Applied Physics, 41, $24-35$.

[3]. Anand Rao, J. and Srinivasa Raju, R., 2010. Applied Magnetic Field on Transient Free Convective Flow of an Incompressible Viscous Dissipative Fluid in a Vertical Channel, Journal of Energy, Heat and Mass Transfer, 32, 265 - 277.

[4]. Anjali Devi, S. P., Shailendhra, K., and Hemamalini, P. T., 2011. Pulsated convective MHD flow with Hall current, heat source and viscous dissipation along a vertical porous plate, Int. J. of App. Math. and Computation, 3(2), 141-150.

[5]. Atul Kumar Singh, Ajay Kumar Singh and N. P. Singh, N. P., 2005. Hydromagnetic free convection and Mass transfer flow with Joule heating, thermal diffusion, Heat source and Hall current, Bulletin of the Institute of mathematics academia sinica, 33(3), $291-310$.

[6]. Chowdhary R. C. and Kumar Jha. A., 2008. Heat and mass transfer in elasticoviscous fluid past an impulsively started infinite vertical plate with Hall Effect, Latin American Applied Research, 38, 17 - 26.

[7]. Chaudhary, R. C., Jain, P., 2007. Hall Effect on MHD mixed convection flow of a Viscoelastic fluid past an infinite vertical porous plate with mass transfer and radiation, Ukr. J. Phys., 52(10).

[8]. Emmanuel Osalusi, Jonathan Side, Robert Harris, Barry Johnston, 2009. On the Effectiveness of Viscous Dissipation and Joule Heating on Steady MHD and Slip flow of a Bingham fluid over a porous rotating disk in the presence of Hall and ion-slip Currents, Romanian Reports in Physics, 61(1), 71 - 93.

[9]. Hossain, M. A., and Rashid, R. I. M. A., 1987. Hall effects on Hydro magnetic Free Convection Flow along a Porous Flat Plate with Mass Transfer, Journal of the Physical Society of Japan, 56, 97 - 104.

[10]. Lai, F. C., 1991. Coupled Heat and Mass Transfer by Mixed Convection from a Vertical Plate in a Saturated Porous Medium, International Journal of Heat and Mass Transfer, 18, $93-106$.

[11]. Maleque, K. A., Sattar, M. A., 2005, The effects of variable properties and hall current on steady MHD laminar convective fluid flow due to a porous rotating disk, International Journal of Heat and Mass Transfer, 48, 4963 - 4972 
[12]. Palumbo, L. J., Platzeck, A. M., 2006, MHD stationary symmetric flows with Hall effect, Journal of Plasma Physics, 72, 457-467

[13]. Rajashekhar, M. N., Anand Rao, J., and Shanker, B., 1999. Numerical Solutions of Hall Effects on Heat and Mass Transfer Flow through Porous Medium, Journal of Energy Heat and Mass Transfer, 21, $1-7$.

[14]. Singh, N. P., 1996. Mass transfer effects on the Flow Past a vertical Porous Plate, Proceedings of Math Soc., 12, $109-114$.

[15]. Singh, N. P., and Kumar, R., 1995. An Integral Treatment for Combined Heat and Mass Transfer by Natural Convention in a Porous Medium, Acta Ciencia India, 21, $451-463$.

[16]. Singh, N. P., Singh, A. K., and Kumar, R., 1996. Free Convection Heat and Mass Transfer along a Vertical Surface in a Porous Medium, Indian Journal of Theoretical Physics, 44, $255-264$.

[17]. Soundalgekar, V. M., Ray, S. N., and Dass, V. N., 1995. Coupled Heat and Mass transfer by Natural Convention from Vertical Surfaces in Porous Medium, Proceedings of Math Soc., 11, 95 - 98.

[18]. Srihari, K., Kishan, N., and Anand Rao, J., 2008. Hall Effect on MHD Flow and Heat Transfer along a Porous Flat Plate with Mass Transfer and Source/Sink,Journal of Energy, Heat and Mass Transfer, 30, 361 - 376.

[19]. Sriramulu, A., Kishan, N., and Anand Rao, J., 2007. Effect of Hall Current on MHD Flow and Heat Transfer along a Porous Flat Plate with Mass Transfer, J. Inst. Eng., 87, 24 - 27. 